\title{
Guided Wave Propagation as a Measure of Axial Loads in Rails
}

\author{
Philip W. Loveday ${ }^{* a}$, Paul D. Wilcox ${ }^{\mathrm{b}}$ \\ ${ }^{a}$ Sensor Science and Technology, CSIR Material Science and Manufacturing, \\ Box 395, Pretoria 0001, South Africa \\ ${ }^{b}$ University of Bristol, Mechanical Engineering, Queen's Building, \\ University Walk, Bristol BS8 1TR, United Kingdom
}

\begin{abstract}
Guided wave propagation has been proposed as a means to monitor the axial loads in continuously welded railway rails although no practical system has been developed. In this paper, the influence of axial load on the guided wave propagation characteristics was analyzed using the semi-analytical finite element method, extended to include axial loads. Forty modes of propagation were analyzed up to a maximum frequency of $100 \mathrm{kHz}$. The sensitivity of the modes to axial load or changes in elastic modulus was formulated analytically and computed. In practice, by using separation of signals in time it would only be possible to separate the mode with the greatest group velocity over a reasonable distance. It was found that the influence of axial load on the wavelength of such a mode should be measureable. However, the influence of changes in the elastic modulus due to temperature is expected to be an order of magnitude larger. In order to develop a practical measurement technique it would be necessary to eliminate or compensate for this and other influences.
\end{abstract}

Keywords: guided wave propagation, rail, axial load measurement, SAFE, semi-analytical finite element method

\section{INTRODUCTION}

Modern railway track consists of rail sections of approximately $25 \mathrm{~m}$, which are welded together to produce lengths of hundreds of meters. This continuously welded rail (CWR) has, in general, no expansion joints and the axial stress in the rail depends on temperature. During installation the rail is pre-tensioned with a tensile load set according to the temperature at the time. The level of tension in the rail can change over time as the track settles after use. A tension larger than desired will accelerate aging of the rail while a too low tension can lead to compression at high temperatures with the possibility of buckling and train derailment. Therefore a technique for measuring the absolute tension in the rail is required. Various measurement techniques are described in [1] but there remains a need for a technique that is easy to implement and that does not interfere with the operation of the railway. It is well known that the resonant frequencies of strings and beams are influenced by the tension or axial load and measurement of wave propagation characteristics have been proposed as a technique for determining the axial load in rail. Damljanović and Weaver $[2,3]$ investigated the use of low frequency $(200 \mathrm{~Hz})$ flexural waves. In this method a scanning laser vibrometer was used to measure the displacements of points along the rail and a technique was used to extract the wavenumber of the flexural mode at 200 Hz. Changes in the wavenumber then reflect changes in the axial load. This method requires that the rail be released from the sleepers for a considerable length. Chen and Wilcox [4] investigated the use of higher frequency guided waves for measuring loads in rods. Simulation results clearly demonstrated that the phase velocity and group velocity are sensitive to changes in load. Although these techniques have been proposed they are not being used in practice as there are still some challenges.

While it is possible to analyze the dispersion characteristics in rails using three-dimensional finite element models [1] this is tedious and very difficult at higher frequencies. An alternative is to use the semi-analytical finite element method, which was recently extended to include axial loads [5]. This allows rapid analysis even at high frequencies.

In this paper we analyze the guided wave propagation in rail subject to axial loads over a large frequency range. We discuss the requirements for a measurement technique based on guided waves and use the numerical results to

*ploveday@csir.co.za

Health Monitoring of Structural and Biological Systems 2010, edited by Tribikram Kundu,

Proc. of SPIE Vol. $7650,765023 \cdot$ C C 2010 SPIE · CCC code: 0277-786X/10/\$18 · doi: 10.1117/12.847531

Proc. of SPIE Vol. $7650765023-1$ 
investigate if such techniques are possible. The sensitivities of the wavenumber to axial loads and to changes in elastic constants are compared.

\section{PRACTICAL MEASUREMENT METHODS}

To exploit guided waves for load measurement it is necessary to make reliable and accurate measurements of either phase or group velocity on real structures. Practically this involves launching a wave packet of a guided wave mode that is sensitive to load at one station and detecting that mode at a second station some distance (the gauge length) away. The objective is to isolate the directly transmitted signal of interest both from all other signals which may include other modes and also from reflections from the end of the structure. The challenge in most practical applications is to obtain an acceptable level of sensitivity to load on the shortest possible structure. Higher sensitivity requires a lower operating frequency but a lower operating frequency means a spatially and temporally longer wave packet which is harder to separate from other signals. A number of approaches for separating signals are possible:

1) Separation of all signals in the time-domain

2) Use of modally selective transduction system to suppress signals from unwanted modes

3) Use of directionally selective transduction system to suppress signals generated or received from unwanted directions.

In practice it is necessary to detect very small changes in the phase or group velocity. While modally and directionally selective transduction systems will reduce the presence of unwanted signals they cannot be expected to completely eliminate these signals. Changes in the amplitudes of these signals could be expected to hide the effects of axial load. In this paper we therefore limit our attention to the option of using separation in the time domain. If a single mode can be separated in time then it has been shown in [4] that the phase velocity within the wave packet is sensitive to axial load as shown in figure 1.
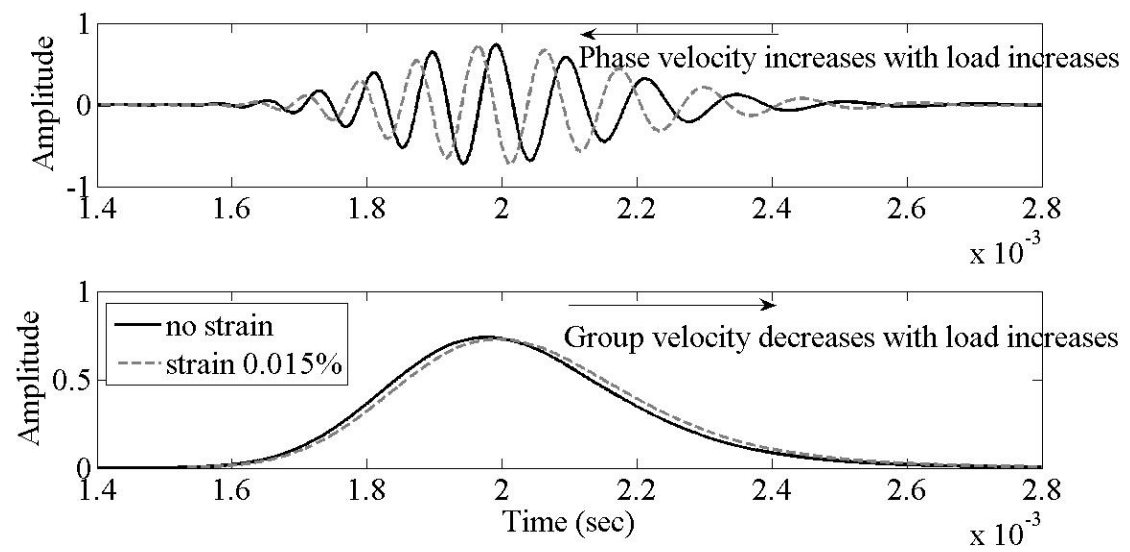

Figure 1. Influence of axial load on (top) phase velocity by consideration of the signal and (bottom) group velocity by consideration of the signal envelope (from [4]).

During the development of a measurement system it is necessary to quantify the sensitivity to the parameter to be measured, in this case the axial load. It is then necessary to quantify the sensitivity to other parameters to ensure that the system will be able to detect the desired change in the presence of other inputs. Optimization of the measurement involves increasing the sensitivity to the desired parameter relative to the undesired parameters. Some practical inputs that such a measurement system must be insensitive to include:

- Changes in elastic constants with time and temperature

- Changes in the support conditions

- Changes in the rail due to re-profiling by grinding.

In this paper we determine conditions for mode separation in time, study the sensitivity of wavenumber to axial load and temperature related changes in elastic constants. 


\section{SAFE WITH AXIAL LOADS}

Chen and Wilcox [1] pointed out that there are three ways in which finite element modelling can be applied to analyse the influence of axial load on waveguides with complex cross-section. Firstly, time domain simulation performed on a three-dimensional model of a length of waveguide may be performed. This is computationally expensive and it is difficult to extract the dispersion data for individual modes of interest. Secondly, a three-dimensional model of a relatively short length of waveguide, with one end restrained in the axial direction, may be used. The axial load is applied by prescribing an axial displacement to the second end of the model and the global stiffness matrix is recomputed before an eigensolution provides natural frequencies and mode shapes of the deformed length of waveguide. The mode shapes are inspected (manually) to determine what fraction of a wavelength and which mode of propagation they correspond to. A few points on the phase velocity curves may then be plotted. The length of the model is then changed and the process is repeated to obtain more points on the phase velocity curves. While this is very tedious Chen and Wilcox demonstrated that it can be applied to a rail, at low frequencies, where there are only a few modes of propagation which are sensitive to axial loads. The third option is to use semi-analytical finite elements, which are specially formulated for modelling the wave propagation in waveguides of arbitrary but constant cross-section. This method is very efficient as only a two-dimensional mesh of the cross-section of the infinitely long waveguide is required. Semi-analytical finite elements are being used by a number of research groups to investigate various wave propagation problems [6-10]. The method was recently extended to include the presence of an axial load and it was shown that the modifications required to a code are trivial [5]. The numerical efficiency of this approach and the fact that the results can be used directly, without significant post-processing by the user, make this approach very attractive for analysing the influence of axial load on the dispersion characteristics of a waveguide.

Gavrić [6] formulated semi - analytical finite elements for modelling the wave propagation in elastic waveguides, with arbitrary but constant cross-section, and applied the method to analyse the wave propagation in rails. These twodimensional elements included a complex exponential function perpendicular to the meshing plane, which represents the wave motion along the waveguide. Similar elements have since been presented by other researchers [7-10] and yield a symmetric eigenvalue problem:

$$
\left[\kappa^{2} \cdot K_{2}+\kappa \cdot K_{1}+K_{0}\right] u=\omega^{2} M u
$$

where $\mathrm{M}$ is the mass matrix, $u$ is the displacement vector, $\omega$ is frequency, $K=\kappa^{2} K_{2}+\kappa K_{1}+K_{0}$ is the stiffness matrix and $\kappa$ is the wavenumber. The mass matrix is derived from the kinetic energy and the stiffness matrix is derived from the strain energy. The application of an initial load results in additional terms in the strain energy which therefore lead to additions to the stiffness matrix. It was shown in [5] that the additional term is proportional to the mass matrix and that the eigenvalue problem, including the initial axial stress, $\sigma_{0}$, is,

$$
\left[\kappa^{2} \cdot\left(K_{2}+\frac{\sigma_{0}}{\rho} M\right)+\kappa \cdot K_{1}+K_{0}\right] u=\omega^{2} M u .
$$

Dispersion characteristics, of propagating modes, may be obtained by solving this eigenvalue problem for a range of different wavenumbers and collecting the real frequencies that are produced. At each wavenumber considered a set of frequencies are obtained and dispersion curves can be extracted based on orthogonality between mode shapes at slightly different wavenumbers [11].

The group velocities can be computed using the analytical expression in (3), which was presented by Hayashi et al. [7] for their element formulation,

$$
v_{g}=\frac{\partial \omega}{\partial \kappa}=\frac{1}{2 \omega} \frac{\psi^{T}\left[2 \kappa\left(K_{2}+\sigma_{0} \frac{M}{\rho}\right)+K_{1}\right] \psi}{\psi^{T} M \psi},
$$


where, $\psi$ is a matrix of eigenvectors.

The starting point for this analytical expression for group velocity is to differentiate the solution to the eigenvalue problem with respect to $\omega$ :

$$
\frac{\partial}{\partial \omega} \psi^{T}\left[\kappa^{2}\left(K_{2}+\sigma_{0} \frac{M}{\rho}\right)+\kappa K_{1}+K_{0}-\omega^{2} M\right] \psi=0,
$$

leading to an expression which can be rearranged to obtain $v_{g}=d \omega / d \kappa$ as the subject. If instead the eigenvalue solution is differentiated with respect to stress, the sensitivity of the wavenumber to axial stress at constant frequency can be obtained directly,

$$
\frac{\partial \kappa}{\partial \sigma}=-\frac{\psi^{T} \kappa^{2} \frac{M}{\rho} \psi}{\psi^{T}\left[2 \kappa\left(K_{2}+\sigma_{0} \frac{M}{\rho}\right)+K_{1}\right] \psi}=-\frac{\kappa^{2}}{2 \omega \rho} \frac{1}{v_{g}}
$$

In a similar way, the sensitivity of the wavenumber to changes in elastic modulus can be obtained and is found to have a similar form,

$$
\frac{\partial \kappa}{\partial E}=-\frac{1}{E} \frac{\psi^{T}\left[\kappa^{2}\left(K_{2}+\frac{M}{\rho} \sigma_{0}\right)+\kappa K_{1}+K_{0}\right] \psi}{\psi^{T}\left(2 \kappa K_{2}+K_{1}\right) \psi}=-\frac{1}{E} \frac{\omega^{2} \psi^{T} M \psi}{\psi^{T}\left(2 \kappa K_{2}+K_{1}\right) \psi}=-\frac{\omega}{2 E} \frac{1}{v_{g}} .
$$

\section{ANALYSIS OF MEASUREMENT OF AXIAL LOADS IN RAILS}

\subsection{SAFE analysis of the influence of axial loads}

A $22 \mathrm{~kg} / \mathrm{m}$ steel rail profile (with Young's modulus, $E=200 \times 10^{9}$ Pa, Poisson's ratio, $v=0.3$ and density $\rho=7700 \mathrm{~kg} \mathrm{~m}^{-3}$ ) was analyzed using linear quadrilateral elements and the mesh shown in figure 2 . Wavenumber and group velocity curves are shown in figures 3 and 4 for no axial load and for an axial load of $200 \mathrm{MPa}$ corresponding to an axial strain of 0.001. The curves are plotted for frequencies up to $100 \mathrm{kHz}$ and a zoomed in view of the low frequency region is provided. It is clear that even this large load has only a small influence on the dispersion curves. The sensitivity of the wavenumber to axial load $\left(\frac{\partial \kappa}{\partial \sigma}\right)$ and the relative sensitivity $\left(\frac{1}{\kappa} \frac{\partial \kappa}{\partial \sigma}\right)$ are shown in figure 5 . The two low frequency flexural waves show the greatest sensitivity but there is considerable relative sensitivity of higher order modes at high frequencies. It should be noted that the sensitivities were only plotted for group velocities greater than $100 \mathrm{~m} / \mathrm{s}$. Equation 4 shows that the group velocity appears in the denominator of the sensitivity expression. The modes cut-on at zero group velocity and therefore infinite sensitivity. Some modes have decreasing group velocity (negative) at first and then the group velocity increases and passes through zero again. These regions were avoided by only plotting larger group velocities. 


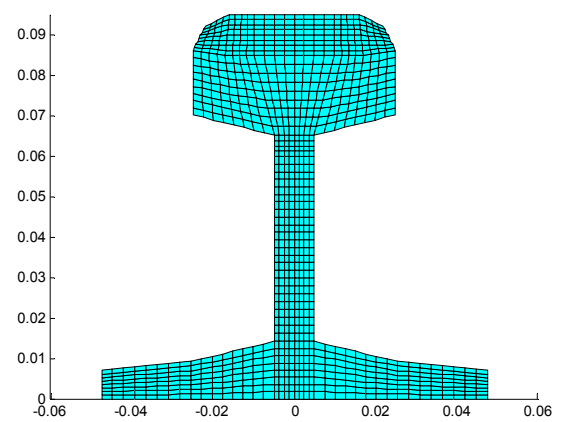

Figure 2. Finite element mesh used in analyses.
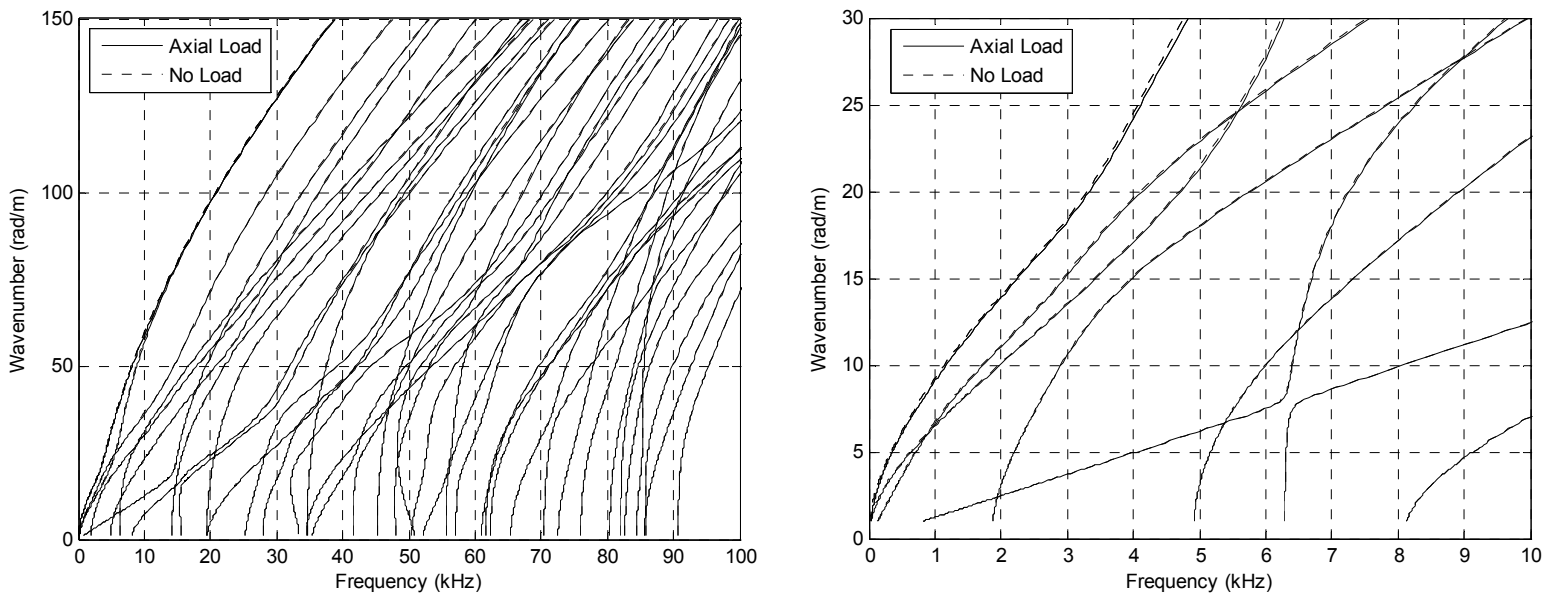

Figure 3. Computed wavenumber - frequency curves with $200 \mathrm{MPa}$ axial load (solid lines) and without axial load (dotted lines).
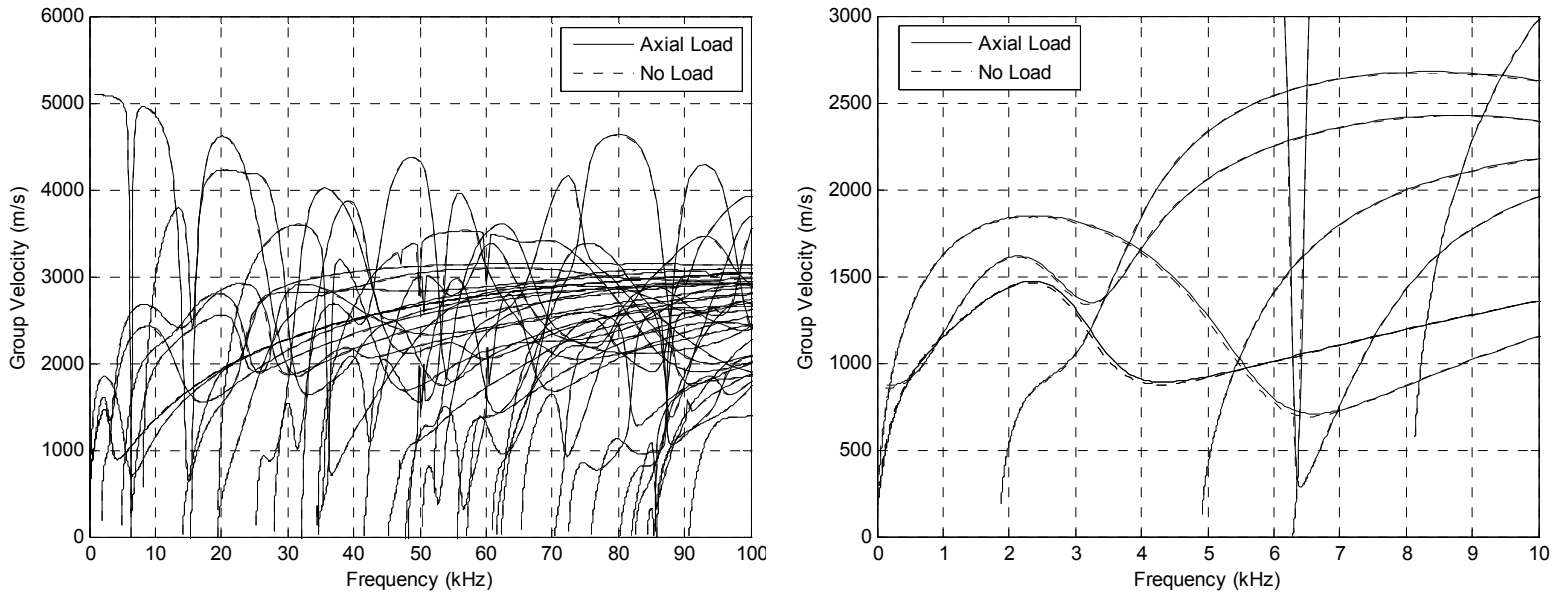

Figure 4. Computed group velocity - frequency curves with $200 \mathrm{MPa}$ axial load (solid lines) and without axial load (dotted lines). 

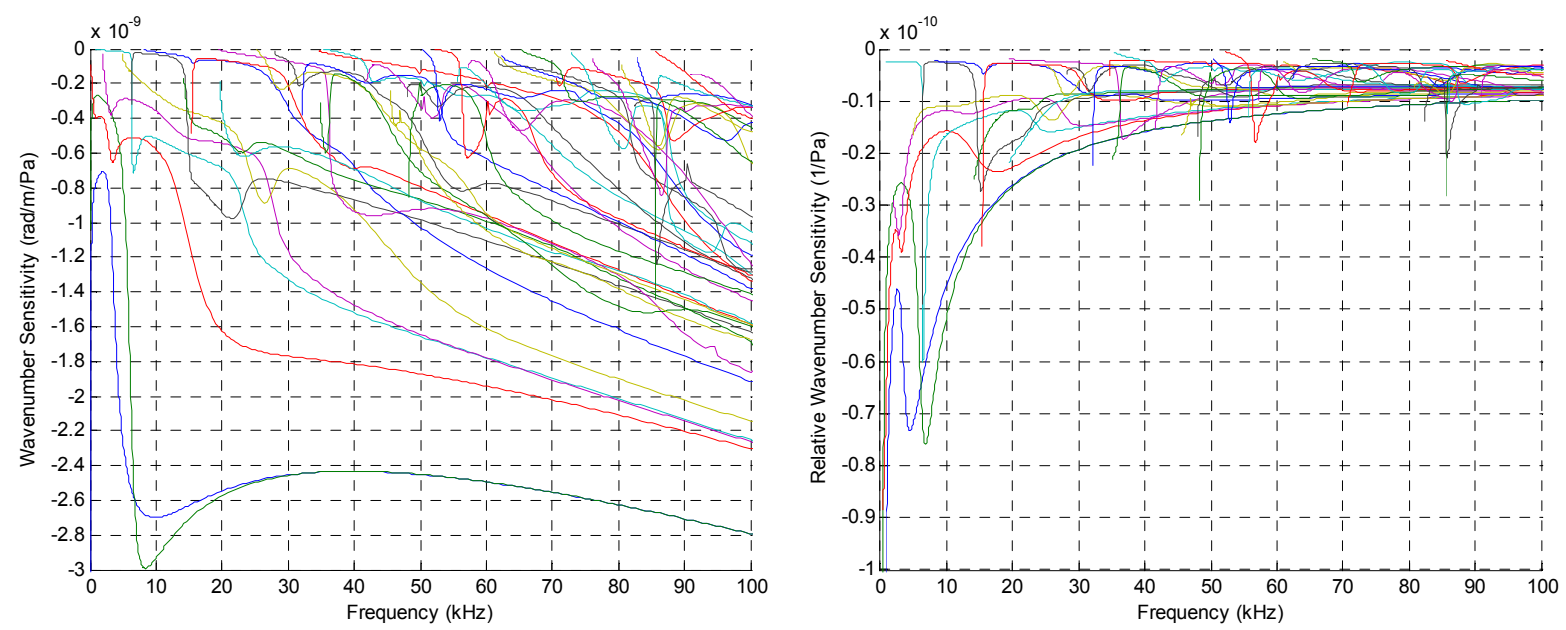

Figure 5. Computed absolute (left) and relative (right) wavenumber sensitivity to axial stress.

\subsection{Implications for axial load measurement}

The group velocity curves reveal that at even fairly low frequencies there are numerous propagating modes with a range of velocities and that many of these exhibit significant dispersion (group velocity is frequency dependent). If we consider a gauge length of $10 \mathrm{~m}$ it is unlikely that any mode other than the fastest one could be separated from the rest in time. If we adopt the strategy of separating the modes in time we then want to quantify the change that can be expected in the first arrival. If we consider a gauge length of $10 \mathrm{~m}$ we can compute the number of wavelengths $(n)$ over this distance $(L)$ with and without the axial load and the change in the number of wavelengths that occurs. This change in the number of wavelengths would appear as a phase shift in the received signal and this is what would provide the measure of the axial load.

$$
n=\frac{L}{\lambda}=\frac{L \kappa}{2 \pi} ; n+\Delta n=\frac{L(\kappa+\Delta \kappa)}{2 \pi} ; \Delta n=\frac{L \Delta \kappa}{2 \pi}
$$

This sensitivity is plotted in figure 6 as the size of the marker on the group velocity curves. It is observed again that the sensitivity is large at small group velocities as explained earlier and that the sensitivity is large for the low frequency flexural modes. The second plot only shows the markers for group velocities greater than $3500 \mathrm{~m} / \mathrm{s}$ as we intend to use a mode with large group velocity. It is seen that the sensitivity increases with frequency because although the change in wavenumber is small, the number of wavelengths in the gauge length increases with frequency. If we consider the mode with the greatest group velocity at $80 \mathrm{kHz}$ we find that an axial stress of $200 \mathrm{MPa}$ causes a change in wavenumber of $0.049 \mathrm{rad} / \mathrm{m}$ which would produce a change in the number of wavelengths over $10 \mathrm{~m}$ of -0.078 or a phase shift of approximately $28^{\circ}$. It is expected that such a phase shift could be measured with reasonable resolution in practice. 

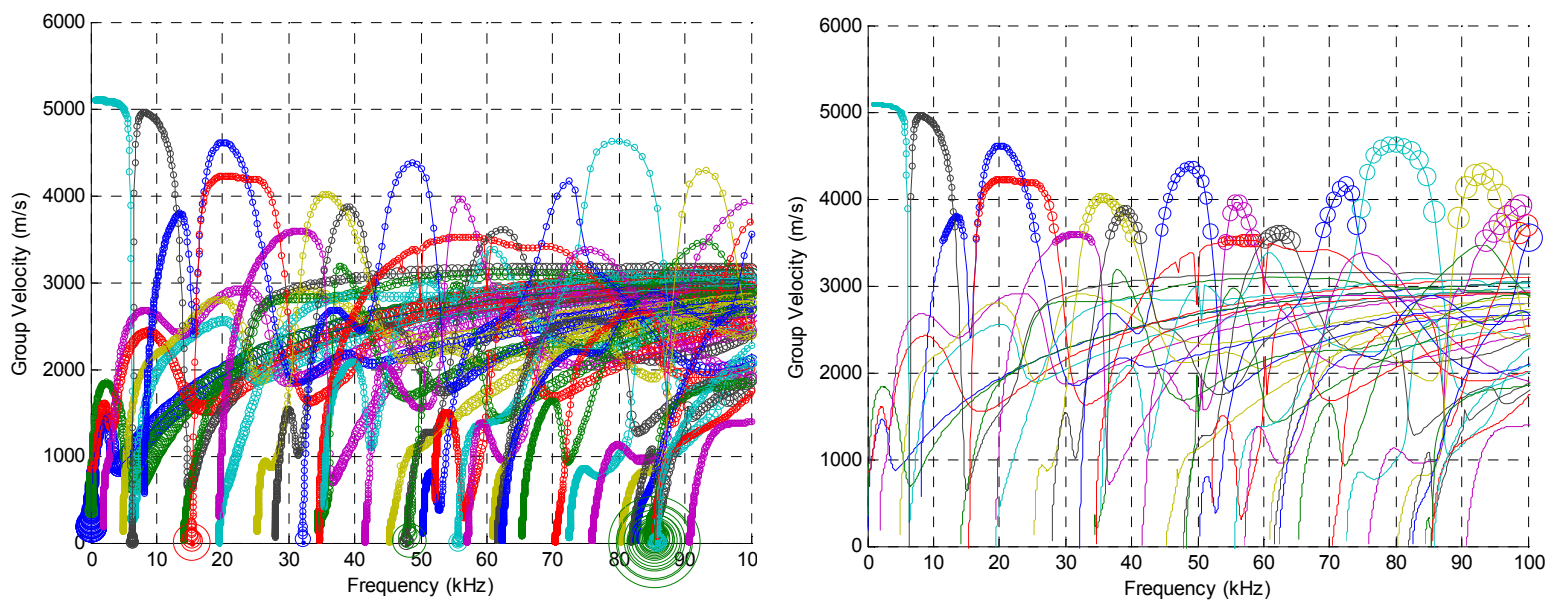

Figure 6. Computed wavenumber shift superimposed on group velocity curves. The size of the circular markers indicates the absolute wavenumber sensitivity at each point. In the right hand graph, only points where the group velocity exceeds $3500 \mathrm{~ms}^{-1}$ are plotted in this way.

\subsection{Analysis of the influence of temperature}

A change in temperature will cause a change in the axial load due to the thermal expansion being constrained. The elastic modulus of the rail will also change with temperature. Both of these mechanisms will cause a change in the wavenumber. This section provides an estimate of the relative sensitivity to these two mechanisms.

Influence of temperature on wavenumber via thermal stress

We assume that the thermal expansion is constrained and results in thermal stress, which can be quantified if we know the thermal expansion coefficient and the Young's modulus.

$$
\sigma(T)=-E \alpha\left(T-T_{0}\right) ; \frac{d \sigma(T)}{d T}=-E \alpha
$$

We can therefore compute the sensitivity of the wavenumber to changes in stress caused by temperature changes.

$$
\frac{\partial \kappa(\sigma)}{\partial T}=\frac{\partial \kappa(\sigma)}{\partial \sigma} \frac{\partial \sigma}{\partial T}=\frac{E \alpha \kappa^{2}}{2 \omega \rho} \frac{1}{v_{g}}
$$

Influence of temperature on wavenumber via elastic modulus

If we assume that the Young's modulus varies linearly with temperature we can estimate the sensitivity of wavenumber to changes in elasticity due to temperature.

$$
\begin{gathered}
E(T)=E_{0}+\frac{E_{1}-E_{0}}{T_{1}-T_{0}}\left(T-T_{0}\right)=E_{0}+\gamma\left(T-T_{0}\right) ; \quad \frac{d E}{d T}=\gamma \\
\frac{\partial \kappa(E)}{\partial T}=\frac{\partial \kappa(E)}{\partial E} \frac{\partial E}{\partial T}=-\frac{\gamma \omega}{2 E} \frac{1}{v_{g}}
\end{gathered}
$$

Comparison of the two effects

As the two sensitivities have the same form it is simple to compare them.

$$
\frac{\partial \kappa(E)}{\partial T}=\frac{-\gamma \omega^{2} \rho}{\alpha E^{2} \kappa^{2}} \frac{\partial \kappa(\sigma)}{\partial T}
$$


We see that the two sensitivities are proportional to each other and note that the phase velocity $(\omega / \kappa)$ should be similar to the longitudinal wave velocity $(\sqrt{E / \rho})$ when we consider large group velocities (in the previous example this ratio was 1.05). Therefore,

$$
\frac{-\gamma \omega^{2} \rho}{\alpha E^{2} \kappa^{2}}=\frac{-\gamma}{\alpha E}\left(\frac{\frac{\omega^{2}}{\kappa^{2}}}{\frac{E}{\rho}}\right) \approx \frac{-\gamma}{\alpha E} .
$$

In order to estimate the ratio of sensitivities to temperature and axial load we assume the following material properties; $E$ $=200 \times 10^{9} \mathrm{~Pa}, \alpha=12 \times 10^{-6} /{ }^{\circ} \mathrm{C} ; \gamma=-0.0002 E[12]$, which yields a value of approximately 17 .

Therefore the wavelength is an order of magnitude more sensitive to elastic modulus changes than axial stress due to temperature changes.

\section{CONCLUSIONS AND RECOMMENDATIONS}

The SAFE method was used to analyze the influence of axial load on the wave propagation in a rail up to $100 \mathrm{kHz}$. It is believed that only modes with the highest group velocity could be separated in time over a reasonable distance. It appears to be theoretically possible to measure the phase shift caused by axial load changes although the sensitivity to changes in elastic modulus due to temperature changes is likely to be an order of magnitude larger. A strategy would have to be devised to eliminate or compensate for this and other practical influences, which have not been quantified. If this can be achieved, it would then be necessary to develop a technique to estimate the absolute axial load rather than only the change in axial load.

\section{REFERENCES}

1. F. Chen, P.D. Wilcox, "The effect of load on guided wave propagation", Ultrasonics 47, 111-122 (2007).

2. V. Damljanović, R.L. Weaver, "Forced response of a cylindrical waveguide with simulation of the wavenumber extraction problem," Journal of the Acoustical Society of America, 115(4), 1582-1591 (2004).

3. V. Damljanović, R.L. Weaver, "Laser vibrometry technique for measurement of contained stress in railroad rail", Journal of Sound and Vibration 282, 341-366 (2005).

4. F. Chen, P.D. Wilcox, "Load measurement in structural members using guided acoustic waves", Annual Review of Progress in Quantitative NDE 25, 1461-1468 (2006).

5. P.W. Loveday, "Semi-analytical finite element analysis of elastic waveguides subjected to axial loads", Ultrasonics 49, 298-300 (2009).

6. L. Gavrić, "Computation of propagative waves in free rail using a finite element technique", Journal of Sound and Vibration 185(3), 531-543 (1995).

7. T. Hayashi, W-J. Song, J.L. Rose, "Guided wave dispersion curves for a bar with an arbitrary cross-section, a rod and rail example", Ultrasonics 41, 175-183 (2003).

8. V. Damljanović, R.L. Weaver, "Propagating and evanescent elastic waves in cylindrical waveguides of arbitrary cross section", Journal of the Acoustical Society of America 115(4), 1572-1581 (2004).

9. I. Bartoli, A. Marzani, F.L. di Scalea, E. Viola, "Modeling wave propagation in damped waveguides of arbitrary cross-section", Journal of Sound and Vibration 295, 685-707 (2006).

10. P.W. Loveday, "Analysis of piezoelectric ultrasonic transducers attached to waveguides using waveguide finite elements", IEEE Transactions on Ultrasonics, Ferroelectrics and Frequency Control 54(10), 2045-2051 (2007).

11. P.W. Loveday, "Simulation of piezoelectric excitation of guided waves using waveguide finite elements", IEEE Transactions on Ultrasonics, Ferroelectrics and Frequency Control 55(9), 2038-2045 (2008).

12. Estimate based on data in table at http://www.engineeringtoolbox.com/young-modulus-d $773 . \mathrm{html}$. 